\title{
FORSA: Exploiting Filter Ordering to Reduce Switching Activity for Low Power CNNs
}

This paper was downloaded from TechRxiv (https://www.techrxiv.org).

\section{LICENSE}

CC BY 4.0

SUBMISSION DATE / POSTED DATE

$12-10-2021 / 14-10-2021$

\section{CITATION}

Halawani, Yasmin; Mohammad, Baker (2021): FORSA: Exploiting Filter Ordering to Reduce Switching Activity for Low Power CNNs. TechRxiv. Preprint. https://doi.org/10.36227/techrxiv.16796134.v1

$\mathrm{DOI}$

10.36227/techrxiv.16796134.v1 


\title{
FORSA: Exploiting Filter Ordering to Reduce Switching Activity for Low Power CNNs
}

\author{
Yasmin Halawani, Member, IEEE, and Baker Mohammad, Senior Member, IEEE
}

\begin{abstract}
Switching activity in digital circuits depends on the temporal distribution of the data participating in the operation which directly influences the interconnect, dynamic power and timing of the system. This paper proposes an efficient method for reducing both power and latency of matrix-multiplication operations found in many applications like convolution neural networks (CNNs). The approach takes advantage of the unique characterizes of CNN with input stationary for efficient multiply-add operation. Since most application use reduce accuracy for MAC, the proposed work assumed 8-bit fixed point representation. As a demonstrator, CIFAR-10 data set has been used for end to end analysis of the filters on a 3-ConV with 2-FC model structure. The filters' were re-ordered to reduce the switching behaviour between successive weight fetching. This directly impacts the dynamic power consumption and miraculously makes the classification pipeline with $20.62 \%$ less power with 32-quantization levels and 8-bit fixed-point representation. Moreover, reduced switching activity leads to reduced interconnect power which is normally high compared to computing. Furthermore, reducing activity reduces cross-coupling capacitance which helps improve timing and noise.
\end{abstract}

Index Terms-Dynamic power, switching activity, CNN, filter, sort, interconnect, efficiency.

\section{INTRODUCTION}

Moore's Law and Dennard scaling have significantly impacted the advancements in computing systems. But with the imminent end of Moore's law and the challenges already faced in power reduction and clock speed-up due to the end of Dennard scaling; energy efficiency has become a prime design constraint [1]. Moreover, near real-time decision making across many critical domains has raised the need for processing information at the resource-constrained edge devices. Artificial Intelligence (AI)-related tasks such as in autonomous vehicles, health care, telecommunications ... etc, are computationally demanding, require high throughput and must be executed with a minimal amount of energy which makes them challenging to be implemented on the edge devices. Convolution neural networks (CNNs) are widely used AI networks in real-time segmentation and classification tasks where the core building block is the matrix-multiplication $(\mathrm{MM})$ as demonstrated in Fig. 1. Moreover, the dynamic power consumption and switching behavior in digital circuits implementing the CNNs are influenced by the distribution of operands fetched from the input and weight matrices. Accordingly, the arrangement of information turns to be an important phase via sorting the elements in the right order for the subsequent step. So the main focus of this work, is to reduce the energy consumption in a CNN by

- Stationary input and sorted filter weight matrix according to the least temporal activity.

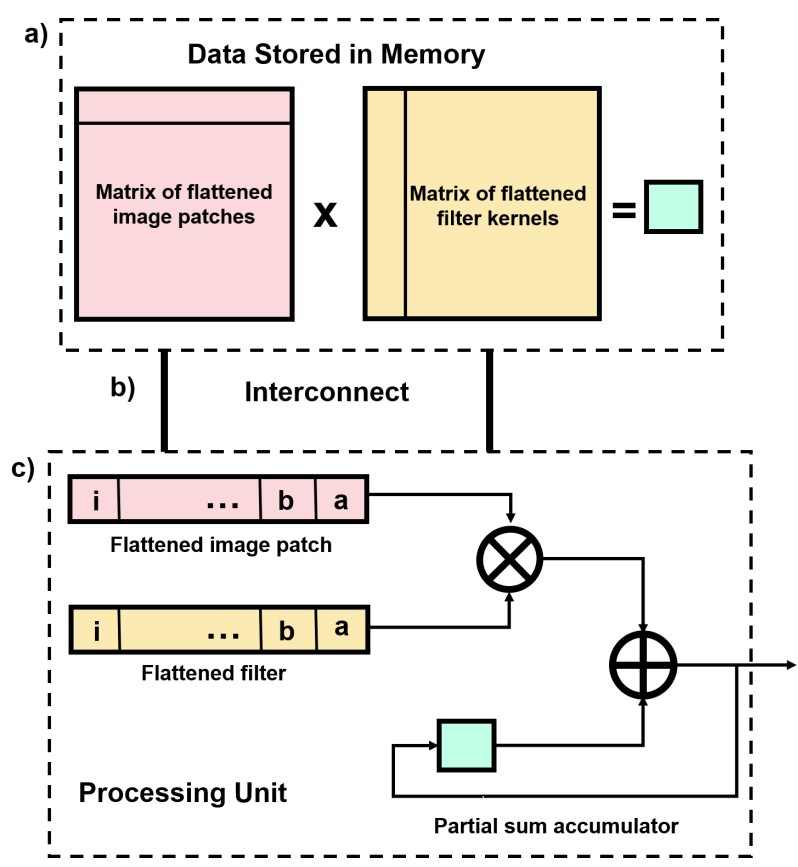

Fig. 1: a) Matrix-multiplication (MM) is the core building block of many applications including neural networks. b) Data operands are fetched from the memory serially and moving to the processing unit through the interconnects. c) Digital implementation of the multiply-add between flattened image patches matrix and flattened filter kernels.

- Analyze the impact of the proposed approach on AI CIFAR-10 and examine the impact on the switching activity and reduced energy consumption.

Major works in the literature on efficient $\mathrm{CNN}$ focus on reducing the complexity through different methods which would reflect on reduced energy, area, and speed-up in performance: Propose techniques to reduce the number of operations: quantization, pruning, fast algorithms, and computational re-use are some examples [2]-[5].

Explore other computing paradigms: In-memory computing (IMC) that is capable of performing computation on the same physical place where data is stored [6]. Such paradigm helps to reduce memory bottleneck and reduce energy and access time.

Exploring new devices: RRAM based IMC paradigm has been extensively used to accelerate the vector matrix multiplication and the convolution operation found in CNN [6]-[8]. RRAM technology has the ability to perform computation over the already stored data by obeying to Ohm's law. Once it is realized in a crossbar structure, it can perform the multipli- 


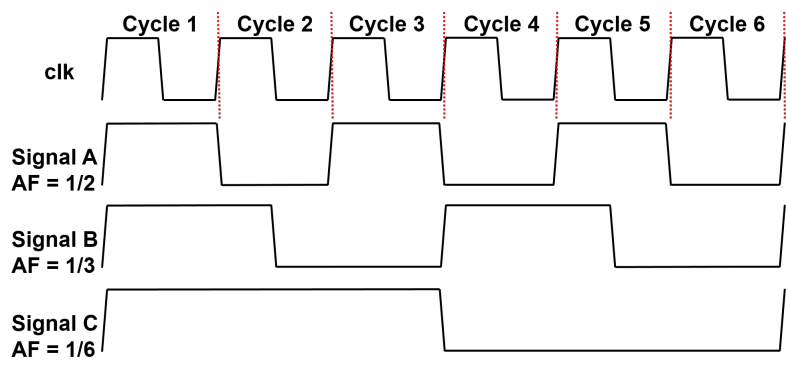

Fig. 2: Timing diagram for different activity factors (AF) illustrates how the switching activity is calculated for different signals with reference to clock.

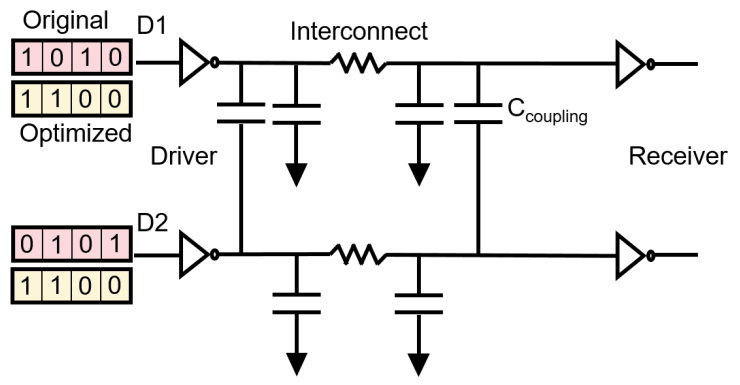

Fig. 3: A CMOS digital design demonstrating how switching activity impacts power, timing and noise. Once a data is arranged in a way to reduce the activity; then both power and noise are reduced as in the second case.

cation and summation in a single shot and thus significantly reducing the number of required MAC units.

Investigate light-weight model designs: SqueezeNet, Mobilenet, ShuffleNet are some examples [9].

Explore other numbering systems: non-conventional computer arithmetic such as the residue numbering system is being used in digital signal processing as a potential for high-speed and parallel computation [10].

Moreover, hardware accelerators for AI grows from the central processing unit (CPU) that can run different algorithms and software but it is not optimized as its a general purpose hardware, to the specialized graphical processing unit (GPU) that is capable of accelerating some parts of the code that can be parallelized. Then to Field-programmable gate array and application-specific integrated circuit designs that can customize the data-flow which is more preferable to accelerate a specific operation while maintaining high throughput. After that, Google's tensor processing unit (TPU) handles MM based on systolic arrays that were built for parallel computing to perform linear algebra of MM. Regardless of the utilized platform to process the application; all share the basic building block MAC unit and off-chip DRAM weight storage but they may differ in the data-flow.

The energy cost of data movement is being dominant in many applications compared to the cost of computations; therefore signal transitions are reduced through an adaptive encoding scheme as in [11]. Moreover, interconnect power can be responsible for over $17 \%$ of the total power budget so in [12], authors propose an on-chip interconnect traffic prediction technique, where future interconnect traffic is predicted from past observations based on an augmentation between the history-table approach with approximate pattern matching to improve prediction accuracy for these patterns. Their proposed approach reduces the average prediction error by around $1.2 \%$. In [13], authors performed time-domain computations with built-in interconnect that can achieve 50\% enhancement in power consumption compared to current techniques. Such method requires time-to-voltage and voltageto-time convertors. Figure 1 presents the MM between the flattened image patches and the flattened filter weight and demonstrates how the two operands are fetched to perform element-wise multiplication and accumulation. Since fetching the operands from the memory and moving data is expensive, several works exploited different data-flow taxonomies such as weight stationary used in TPUs, row stationary and no local re-use [14].

The rest of the paper is organized as follows: Section II introduces the re-ordering method with input stationary for energy efficient MAC computation. The simulation results are presented in Section III. Section V concludes the paper and highlights some future-work points.

\section{Proposed Re-ordering Method}

Dynamic power consumption in CMOS digital design can be calculated through the following

$$
P_{\text {dynamic }}=\alpha C V^{2} f,
$$

where $\alpha$ is the switching activity, $C$ is the load capacitance, $V$ is the supply voltage, and $f$ is the clock frequency. The switching activity $\alpha$ is the probability a given node will change from 1 to 0 or vice versa at a given clock tick and is a function of data patterns between 2 consecutive clock cycle signals. When two successive inputs are re-arranged depending on how similar they are to each other; the switching activity is reduced as shown in Fig. 2. And as for the CNN, changing the order of features has no impact on classification as the filter features are considered mutually independent [15]. Moreover, Fig. 3 shows original vs optimized fetching of data. The worst case where data transmit is different in every consecutive cycle and incur worst power and noise, while in the second case when data is re-arranged then both power and noise can be saved [16].

Figure 4 illustrates the flow-chart of the proposed method. Starting from block (1), a CNN consisting of 3-ConV with 2FC structure was trained using Caffe deep learning framework on CIFAR-10 data-set until the output classification results converged to $75.69 \%$. Then in block (2), the whole spectrum of the weights in each layer was quantized into a certain number of quantization-levels (QLs) 16, 32,and 64. After that, precision reduction was performed on all quantized pre-trained weights from 32-bit floating-point (FP) into 8-bit fixed-point (FI) representation to help limit the incurred overheads as power cost of FP hardware prohibitively expensive in block (3). Classification accuracies are reported for all $\mathrm{CNN}$ data types and QLs in Table II. Next, all filters weights will 


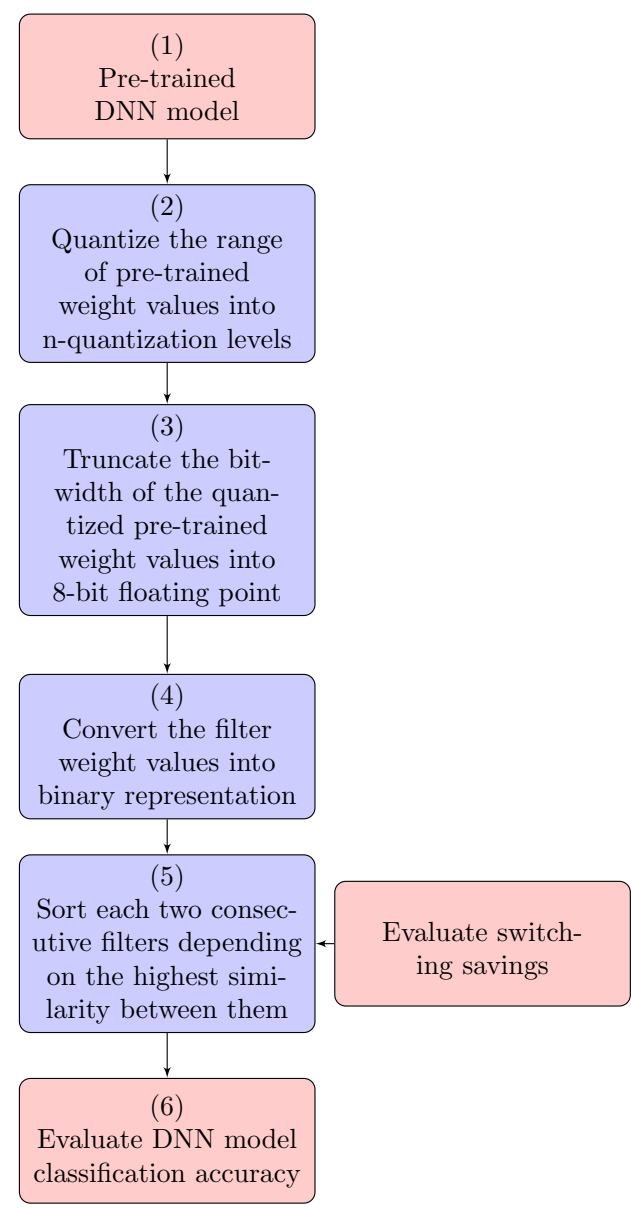

Fig. 4: Flowchart explaining the proposed method of stationary input and sorted filter weights.

be converted into binary representation as block (4) shows. Table I shows a sample part of the filters and how they got sorted. In our CNN model, ConV1 contains 3 channels $(\mathrm{R}, \mathrm{G}, \mathrm{B})$ with 32 filters in each. Initially, the pre-trained filters will flow consecutively as $\mathrm{f} 1, \mathrm{f} 2, \mathrm{f} 3, \ldots, \mathrm{f} 31, \mathrm{f} 32$. A bit-wise comparison is performed between the elements in the first filter column and all proceeding ones. The filter with the highest similarity swaps its location with the second filter. So when $\mathrm{f} 2$ is fetched after $\mathrm{f1}$, minimum number of bits will switch causing less power dissipation. Then, f1, new f 2 are fixed and all upcoming filters are compared with the new $\mathrm{f} 2$ to compare and swap. The sample in Table I in the after sorting column, shows that $\mathrm{f} 2$ was the highest similarity to $\mathrm{f} 1$ and hence did not swap its location. While f3 got swapped with f21. This is captured in block (5).

Figures 5 and 6 demonstrates the features of CIFAR-10 on 3ConV-2FC model for $\mathrm{R}$ channel in ConV1 layer with 32-QLs and 8-bit FI before and after re-arranging the filters. It can be noticed that the consecutive sorted filters are visually similar.

\section{Simulation Results}

Inference phase simulation on the 3-ConV with 2-FC CNN model was implemented on MATLAB R2019b to perform the quantization, truncation, and sorting. Table II

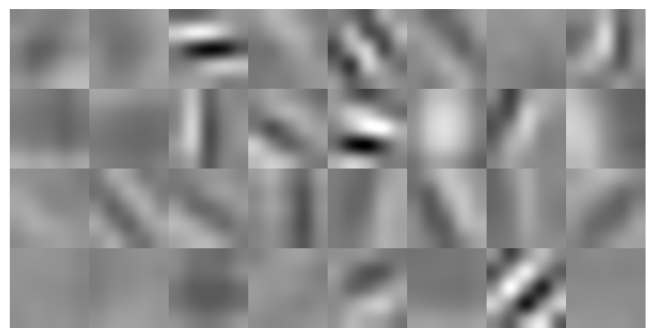

Fig. 5: Visualize Features of CIFAR-10 on 3ConV-2FC Model before sorting for ConV1 R-channel 32-QLs.

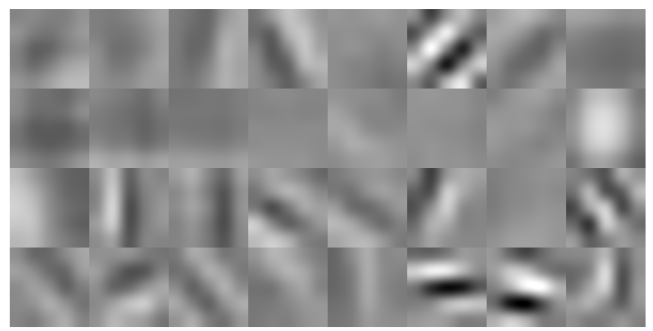

Fig. 6: Visualize Features of CIFAR-10 on 3ConV-2FC Model after sorting for ConV1 R-channel 32-QLs.

displays the simulation results for the three different QLs and compared to the 8-bit FI. A layer-wise switching savings is shown along with the full CNN pipeline savings. The savings depend on the distribution of data and the number of unique weights available after quantization and bit truncation. As the number of unique elements is increased; the savings in switching becomes less as we need to switch more. A detailed MATLAB code is available at GitHub: https://github.com/YasmineHalawani/FORSAExploiting-Filter-Ordering-to-Reduce-Switching-Activity-forLow-Power-CNNs/upload.

Table III shows the participation of switching from each layer to the whole network before sorting, which was calculated by

$$
\text { Layer contribution }=\frac{\text { Layer switching }}{\text { Total switching }} \times 100,
$$

While for the after sorting column, the values were calculated by

$$
\text { Layer contribution }=\frac{\text { Layer savings }}{\text { Total switching }} \times 100,
$$

where Layer switching is the total number of switching occurring in a certain layer, Layer savings is the total number of switching savings gained in a certain layer, and Total switching is the total number of switching in the whole pipeline. And it can be deduced that for the chosen network model, ConV3 participates the most in switching and in savings. That is because not only the size of the weight matrix that matters, but also the number of unique elements and the way quantization was performed. In our work, quantization was performed per channel, ConV3 has 32 channels with a dimension of $5 \times 5 \times 64$, while FC2 has one channel with $1025 \times 64$ elements. 
TABLE I: Partial Sample of the Binary Representation of Filters Before and After Sorting

\begin{tabular}{|c|c|c|c|c|c|c|c|c|c|c|c|}
\hline \multicolumn{6}{|c|}{ Before } & \multicolumn{6}{|c|}{ After } \\
\hline f1 & f2 & f3 & $\cdots$ & f 21 & $\ldots$ & f1 & $\mathrm{f} 2$ & $\mathrm{f} 21$ & $\ldots$ & f3 & $\ldots$ \\
\hline '11111111' & '11111110' & '11111101' & & '11111110' & & '11111111' & '11111110' & '11111110' & & '11111101' & \\
\hline '00000001' & '11111110' & '00000111' & & '11111110' & & '00000001' & '11111110' & '11111110' & & '00000111' & \\
\hline '11111111' & '00000000' & '11111110' & & '11111110' & & '11111111' & '00000000' & '11111110' & & '11111110' & \\
\hline '11111111' & '00000001' & '11111110' & & '11111110' & & '11111111' & '00000001' & '11111110' & & '11111110' & \\
\hline '00000001' & '00000001' & '00000001' & $\ldots$ & '11111110' & $\ldots$ & '00000001' & '00000001' & '11111110' & $\ldots$ & '00000001' & $\cdots$ \\
\hline '00000000' & '11111110' & '11111101' & & '11111110' & & '00000000' & '11111110' & '11111110' & & '11111101' & \\
\hline '11111110' & '11111110' & '00001010' & & '11111101' & & '11111110' & '11111110' & '11111101' & & '00001010' & \\
\hline '11111100' & '11111 & '11111 & & '1111 & & '111 & '111 & '111 & & 000' & \\
\hline '11111101' & '11111110' & '11111111' & & '11111101' & & '11111101' & '11111110' & '11111101' & & '11111111' & \\
\hline '00000000' & '00000000' & '00000001' & & '11111110' & & '00000000' & '00000000' & '11111110' & & '00000001' & \\
\hline
\end{tabular}

TABLE II: Switching Savings Layer-wise after Sorting the Fixed Weight Matrices for 16, 32, and 64-QLs. Baseline accuracy $=75.69 \%$ (32-floating-point).

\begin{tabular}{|l||c||c||c||c|}
\hline & 16-QLs & $32-\mathrm{QLs}$ & $64-\mathrm{QLs}$ & 8 -bit fixed-point \\
\hline $\begin{array}{l}\text { Classification } \\
\text { Accuracy }\end{array}$ & $60.54 \%$ & $71.47 \%$ & $71.13 \%$ & $74 \%$ \\
\hline $\begin{array}{l}\text { Total savings } \\
\text { (all layers) }\end{array}$ & $21.01 \%$ & $20.62 \%$ & $20.56 \%$ & $20.51 \%$ \\
\hline ConV1 & $29.9 \%$ & $31.4 \%$ & $30.8 \%$ & $29.77 \%$ \\
\hline ConV2 & $34.5 \%$ & $35.2 \%$ & $34.7 \%$ & $34.51 \%$ \\
\hline ConV3 & $37 \%$ & $36.7 \%$ & $36.5 \%$ & $36.94 \%$ \\
\hline FC1 & $4.3 \%$ & $3.9 \%$ & $4 \%$ & $3.81 \%$ \\
\hline FC2 & $10.6 \%$ & $8.5 \%$ & $10.6 \%$ & $8.32 \%$ \\
\hline
\end{tabular}

TABLE III: Portion of the CNN layer from the total model pipeline before and after sorting for 32-QLs with 8-bit FI representation.

\begin{tabular}{|l||c|c|}
\hline \multicolumn{1}{|c||}{} & \multicolumn{2}{c|}{$32-\mathrm{Q} 1 \mathrm{~s}$} \\
\hline & Before & After \\
\hline ConV1 & $1.68 \%$ & $0.53 \%$ \\
\hline ConV2 & $16.9 \%$ & $5.96 \%$ \\
\hline ConV3 & $33.37 \%$ & $12.25 \%$ \\
\hline FC1 & $47.6 \%$ & $1.86 \%$ \\
\hline FC2 & $0.43 \%$ & $0.037 \%$ \\
\hline
\end{tabular}

\section{CONCLusions AND Future Work}

Power is one of the prime constraints in the electronics design especially for the emerging $\mathrm{AI}$ and big data era. Moreover, dynamic power consumption in digital design is directly impacted by the switching activity of the node from 1 to 0 or vice versa. The switching behaviour itself depends on the distribution of the operands participating in the operation. This paper takes advantage of the fixed filter weights during inference phase. The proposed work shows that re-ordering of these weights helps reduce the switching activity and as a result active power from both interconnect and gate is also decreased. In addition, it improves timing by eliminating the cross coupling capacitance. A reduction of $20.62 \%$ in switching activity for 32-quantization levels and 8-bit fixedpoint representation is obtained. In future, the impact on timing and memory access can be discussed.

\section{ACKNOWLEDGMENT}

This publication is based upon work supported by the Khalifa University Competitive Internal Research Award (CIRA) under Award No. [CIRA-2019-026] and System-on-Chip Center Award No. [RC2-2018-020].

\section{REFERENCES}

[1] L. Johnsson and G. Netzer, “The impact of Moore's law and loss of Dennard scaling: Are DSP SoCs an energy efficient alternative to x86 SoCs?," in Journal of Physics: Conference Series, vol. 762, p. 012022, IOP Publishing, 2016.

[2] Y. Halawani, B. Mohammad, and H. Saleh, "Design exploration of ReRAM-based crossbar for AI inference," IEEE Access, vol. 9, pp. 70430-70442, 2021.

[3] T. Abtahi, C. Shea, A. Kulkarni, and T. Mohsenin, "Accelerating convolutional neural network with FFT on embedded hardware," IEEE Transactions on Very Large Scale Integration (VLSI) Systems, vol. 26, no. 9, pp. 1737-1749, 2018.

[4] J.-H. Luo, H. Zhang, H.-Y. Zhou, C.-W. Xie, J. Wu, and W. Lin, "Thinet: pruning CNN filters for a thinner net," IEEE transactions on pattern analysis and machine intelligence, vol. 41, no. 10, pp. 2525-2538, 2018.

[5] F. Alantali, Y. Halawani, B. Mohammad, and M. Al-Qutayri, "SLID: Exploiting spatial locality in input data as a computational reuse method for efficient CNN," IEEE Access, vol. 9, pp. 57179-57187, 2021.

[6] Y. Halawani, B. Mohammad, M. Abu-Lebdeh, M. Al-Qutayri, and S. F. Al-Sarawi, "ReRAM-based in-memory computing for search engine and neural network applications," IEEE Journal on Emerging and Selected Topics in Circuits and Systems, 2019.

[7] Y. Wang, L. Xia, T. Tang, B. Li, S. Yao, M. Cheng, and H. Yang, "Low power convolutional neural networks on a chip," in IEEE International Symposium on Circuits and Systems (ISCAS), pp. 129-132, 2016.

[8] X. Peng, R. Liu, and S. Yu, "Optimizing weight mapping and data flow for convolutional neural networks on RRAM based processing-inmemory architecture," in IEEE International Symposium on Circuits and Systems (ISCAS), pp. 1-5, 2019.

[9] X. Zhang, X. Zhou, M. Lin, and J. Sun, "Shufflenet: An extremely efficient convolutional neural network for mobile devices," in Proceedings of the IEEE Conference on Computer Vision and Pattern Recognition, pp. 6848-6856, 2018.

[10] L. Sousa, "Nonconventional computer arithmetic circuits, systems and applications," IEEE Circuits and Systems Magazine, vol. 21, no. 1, pp. 640, 2021.

[11] E. Maragkoudaki and V. F. Pavlidis, "Energy-efficient time-based adaptive encoding for off-chip communication," IEEE Transactions on Very Large Scale Integration (VLSI) Systems, vol. 28, no. 12, pp. 2551-2562, 2020.

[12] V. Adhinarayanan and W.-c. Feng, "Approximate pattern matching for on-chip interconnect traffic prediction," in Proceedings of the ACM International Conference on Parallel Architectures and Compilation Techniques, pp. 357-358, 2020.

[13] A. D. Hossain, C. Ni, Q. Sharar, M. Hossain, et al., "Time-domain arithmetic logic unit with built-in interconnect," IEEE Transactions on Very Large Scale Integration (VLSI) Systems, vol. 25, no. 10, pp. 2828$2841,2017$.

[14] V. Sze, Y.-H. Chen, T.-J. Yang, and J. S. Emer, "Efficient processing of deep neural networks: A tutorial and survey," Proceedings of the IEEE, vol. 105, no. 12, pp. 2295-2329, 2017.

[15] A. Sharma, E. Vans, D. Shigemizu, K. A. Boroevich, and T. Tsunoda, "Deepinsight: A methodology to transform a non-image data to an image for convolution neural network architecture," Scientific reports, vol. 9, no. 1, pp. 1-7, 2019.

[16] N. H. Weste and D. Harris, CMOS VLSI design: a circuits and systems perspective. Pearson Education India, 2015. 